\title{
Headway generation with ROBERTO
}

\author{
A. D. Middelkoop \\ ProRail Rail Development, The Netherlands
}

\begin{abstract}
A traditional and expensive solution for bottlenecks in a railway network is to build more infrastructure. To handle future growth of passenger and freight transport demand, the Dutch rail infra manager ProRail is looking for alternative ways to solve capacity and quality bottlenecks. One of the ideas is to evaluate and improve the timetable development process. By applying design principles and by other conditions, buffer times are used in different timetable construction phases. It is not clear whether and where the use of buffer times may cause a double claim on capacity. An important design principle is the use of headway times to separate two trains in the timetable safely. The specific values for headway situations are mostly unknown. Planners use general values, based on their experience. The current timetable planning tools require headway times as input data. Given the large number of potential train combinations, it is almost impossible to know every headway time before timetable construction starts. To improve the knowledge and application of headway times, ProRail started the development of ROBERTO, a tool for generating a large number of headway times. The aim is to compute headway times for specific situations and to determine general headway times for use on a more global level. The input for ROBERTO is generated by simulating train characteristics, block section occupation times and signalling aspects. ROBERTO combines possible conflicting train pairs and calculates the headway times. All results are fed into the planning systems and the effect on capacity and quality will be evaluated.
\end{abstract}

Keywords: timetable design, headways, simulation.

\section{Introduction}

This paper describes the development of a new planning support tool ROBERTO, for generating a large number of headway times. After a short introduction of the Dutch Rail Network and its challenge for the near future in 
Section 2, it explains the programme of Triple $\mathrm{C}$ in Section 3. This programme consists of a number of measures, which amongst others change the planning and the operation principles of the railway traffic. A particular part is to reallocate buffer times in the timetable. Therefore, historical and experience based headway times are not accurate enough and there is a need to improve the quality of headway times and to make them available in the timetable development and planning systems. Section 4 describes the approach to develop the new tool, Section 5 describes the first application on an important railway corridor in the network. The paper ends with conclusions and future activities

\section{The Dutch rail network}

The size of the infrastructure of the Dutch railway network is moderate compared to most other European countries. The length is $2800 \mathrm{~km}$ and it contains about $6500 \mathrm{~km}$ of tracks. In recent years three new infrastructure lines have been built. A new high speed line Amsterdam-Schiphol-RotterdamBelgium, a new connection between the harbour of Rotterdam and Germany (Betuweroute) and a new extra double track connection between Amsterdam and Utrecht will become available. Anticipating the availability of these capacity extensions ProRail and the Train Operating Companies (TOC) have introduced a new timetable structure in 2007. The main part of the traffic is used for passenger transport (about 85\%). On a daily basis some 5400 trains carry 1.2 million passengers. Each day over 300 cargo trains transport 100 kton of freight. All trains from 29 TOC's produced over 140 million train kilometres in 2007. Although freight transport is growing strongly, it is still a minor part (about 8\%) of the total train kilometre performance.

\subsection{Travelling in the near future}

In the near future Dutch society is facing a mobility problem. Transport demand is expected to increase. It will be difficult to reach city centres, main harbours and to establish good connections to the rest of Europe. Both passenger and freight transport might encounter loss of travel times. In addition, the railway transport demand will grow strongly, especially in the western and most urbanised part of the network (Randstad), as a result of the new timetable structure, the operation of the new lines and road congestion.

Nowadays the occupation rate of the Dutch rail network is already high [1]. In the Randstad intercity trains run every 15 minutes there and regional trains run every 15 minutes, connecting the four largest cities. It will be difficult to facilitate future growth. A traditional and expensive solution for bottlenecks in a railway network is to build more infrastructure. The challenge is to find solutions that are more cost effective.

ProRail, the Dutch rail infra manager has the ambition to facilitate this growth and contribute to the improvement of mobility and reachability. Therefore, ProRail has introduced a programme called Triple C. The idea is to increase railway transport by offering high frequent travel opportunities and to give 
freight lines dedicated routes through the country. There are a number of bottlenecks to solve before the network may handle the foreseen transport volumes.

The ambition is to be ready in 2012 and apply the principles on a first corridor. Depending on the market demand soon other corridors may follow. New solutions from the Triple $\mathrm{C}$ approach will be introduced the coming years as soon as possible.

\section{Triple C project}

The project Triple C, where C stands for 'Change' (in Dutch Triple A), aims on changing or redesigning the timetable development processes. It covers capacity analysis, timetable development and operational processes. A multidisciplinary design team investigates how these processes may be changed to facilitate improvements. Furthermore, they look at conditions concerning maintenance, traffic control, safety, noise, environmental, legal and regulation issues.

This new approach tries to increase transport capacity in a cheaper, smarter and quicker way and focuses on tailor made but robust solutions. It looks for ways to improve the occupation rate first before deciding to build new infrastructure. It also aims at developing innovative solutions and to organise a strong feedback from the operational level to the planning. The next section illustrates the three categories with some examples.

\subsection{Changing capacity extension}

The measures focus on increasing track occupation rates, rail infrastructure extension and river crossings. Examples are:

- Signalling block shortening

- Higher passing speed for freight trains in a node

- Fast overtaking situations

- Opening times waterway bridges

- Alternative waterway crossings

- Advanced traffic management systems to optimise train traffic on punctuality, energy consumption and throughput.

\subsection{Changing capacity allocation}

The infra manager is responsible for the optimal use of the rail and transfer capacity. In case of conflicting capacity claims of different transport companies, this should be the criterion to decide on the final timetable construction. Therefore example measures in this category are:

- New or adapted regulation

- Priority rules for capacity allocation

- Adjust product specification like train types and train lines

- New braking regulations 


\subsection{Changing planning and operational processes}

Timetable planners use planning principles in order to construct a timetable that is feasible during operation. In reality, the structural feedback from the operation to the planning is rather new, but first results are very promising. It triggers a discussion about changing planning principles. Concept measures from this category are:

- Compute and reallocate headway and buffer times

- Small conflicts in planning handled by traffic control

- Minimise crossing movements in big nodes

- $\quad$ Flexible departure tracks

- Support driver and traffic control with on-line information

- Maintenance inspection outside the rush hour

- Quicker door closing process

One of the ideas from the last category is to determine the amount of buffer time in the timetable explicitly and to reallocate the buffer times. By knowing the technical minimal times exactly, the planning process becomes more transparent. The effects of buffer time reallocation and small planning conflicts on capacity and quality may be quantified better. For a lot of situations headway and buffer times are not available on an appropriate level and it is very time consuming to compute them for all situations. This has been the reason to start the development of a tool that generates a large amount of headway times: ROBERTO.

\section{Improving the planning process}

First, this chapter gives an overview of the planning process at ProRail, including the decision support systems. The application of these tools has evolved from not only supporting long term timetable development to also support short term timetable design processes. They are used at ProRail, the Dutch rail infrastructure manager, and at NSR, the main Dutch train operating company. [5]. Then it describes which part is improved by the introduction of ROBERTO.

\subsection{Planning process and tools}

The mid term planning process at ProRail consists of four stages. After these stages, the planning process continues with the construction of the 24-hour timetable, rolling stock, shunting and crew planning. Finally, the capacity allocation process integrates all train paths for all train operating companies.

The first stage is a definition of the transport demand in terms of passenger and freight volumes, train lines, frequencies and more. The second stage is to make a definition of the expected capacity. In fact, this is a description of the infrastructure of the rail network, with an appropriate level of details for the stations and the tracks. The properties of the signalling system are incorporated by means of headway times. The third stage is to generate a countrywide feasible timetable using the CADANS-algorithm [2], which is incorporated in the DONS- 


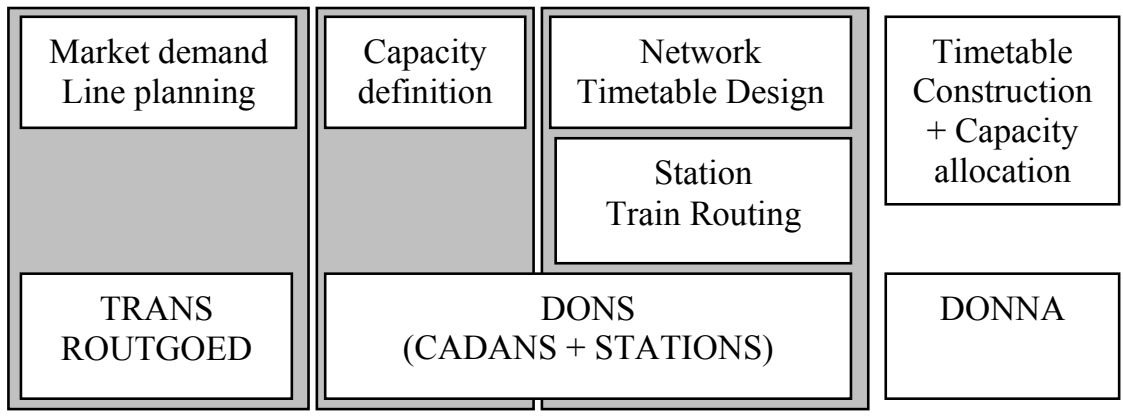

Figure 1: $\quad$ Planning stages and tools.

tool $[4,5]$. In the Netherlands, such a timetable is a regular one or two hourly pattern representing a rush hour or an off-peak period. Also known as clockface pattern timetable or Taktfahrplan in German. The result is a set of records in which each train has an arrival, departure or pass through movement, at a station on a certain moment. The last step is to generate a feasible set of routes in the main stations (about 70) given the network timetable, resulting in track occupation charts. This stage uses the STATIONS algorithm [3].

\subsection{DONS approach: designing a network timetable}

The system DONS (Designer of Network Schedules) supports the construction of a network timetable. It translates the user input into groups of constraints, describing the relation between train events caused by running times, dwell times, headway times buffer times, passenger and rolling stock connections and generates a solution. The result is a set of records where each train has an arrival, departure or pass through movement, at a station on a certain moment. The user has option to give the solver a lot of solution space or to limit this. For instance, when the infrastructure constraints are switched off any timetable should be possible. If in this case no solution is found the market demand is inconsistent and should be changed. The system gives information which set of relations is impossible. On the other hand, the user may also start with an earlier found solution, fix the train times and ask the system to show whether new trains fit in the timetable. If not, the result of former iterations is saved and only new trains should be changed. It is an iterative approach where the tool generates feasible solutions or shows where planners should relax constraints to solve infeasibility.

A feasible timetable means that there are no planning conflicts. Potential conflicts occur amongst others where two trains claim the same infrastructure elements in their path through the network. Most planners and also their timetable planning systems use a microscopic infrastructure model, including switches and the signalling system, to check for conflicts. The planned times are based on a technical minimum time added with a buffer time and rounded to minutes. The underlying network infrastructure model in DONS is on a mesoscopic level. Main elements are the tracks in the nodes, the links and how 
they are connected. It does not describe the switches and the signals, but separates succeeding and crossing train movements by headway times. Unless it is possible to specify a headway situation for a unique train combination in DONS, planners use more general definitions based on their experience and scarce historical facts. The origin and the exact values for underlying headway situations are mostly unknown. The calculation of a headway time is very time consuming, mainly caused by the manual input of infrastructure data. Given the large number of potential train combinations, it is almost impossible to know all correct headway times before timetable construction starts. This number may even grow when more variants of rolling stock combinations should be used. Therefore, in practice these planning norms are simplified to one or more general levels.

With the tool ROBERTO it is possible to generate a large number of headway times automatically and to feed them into DONS and other the planning systems. The aim is to investigate whether simplification to a more general level makes sense and to decide where to use specific or general headway times.

\section{Development of ROBERTO}

To improve the quality of the headway times and to base planning standards on real facts, ProRail started the development of ROBERTO (in Dutch this is an abbreviation of running and headway times calculation tool). The tool supports the calculation of a large number of headway times automatically. This section explains the elements of a headway calculation first, then it describes the ROBERTO development.

\subsection{Calculation of a headway time}

Headway times describe the time distance between two trains in the timetable planning. Each train needs a free track section ahead to guarantee a safe train run. Due to the low adhesive power of the wheel rail contact (steel to steel) and the condition to have an absolute braking distance to any preceding train, braking distances in railways are relatively long compared to road transport. This distance has to be free of other trains in case of normal operation. The signalling system secures safe access to the required infrastructure ahead of a train by showing red, green or yellow aspects sometimes accompanied by a speed limit.

To explain the elements that contribute to a headway time, Figure 2 shows a situation where the location of the conflicting infra element lies outside the platform area. The platform area, represented by a central line $\mathrm{P}$, is the reference location for the moments recorded in the timetable. This is a situation where an arriving train 2 has to be separated from a departing train 1 . The same principles hold for other combinations of arriving, departing and passing trains.

Basic elements in a headway situation are:

- Operation time for setting the route of train 1

- Reaction time driver 


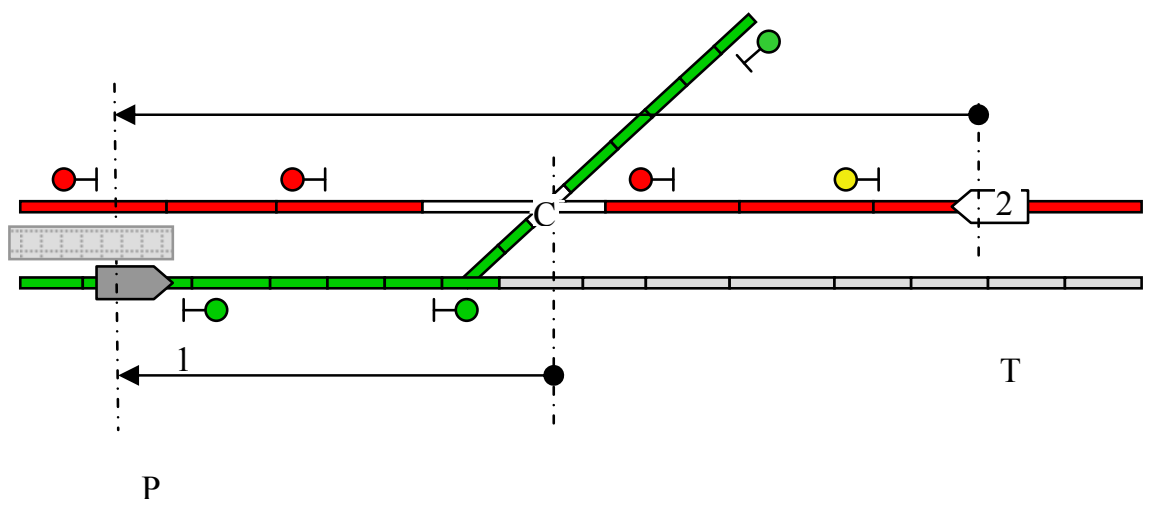

Figure 2: Headway situation.

- Running time train 1, from reference location $\mathrm{P}$ (here platform area) to point that releases the conflicting block section $\mathrm{C}$.

- operation/reaction time to set a new route for train 2over the conflicting element

- Running time train 2, from position $\mathrm{T}$, which is at approaching distance of the relevant signal to platform area $\mathrm{P}$

When two trains share one or more infrastructure elements in their routes, a conflict may occur in case they claim for use this element in an overlapping time interval. Train separation may be described using the blocking time model [6]. In a time-distance graph, a train path is visualised by a line, but on a more detailed level it consists of a set of block section occupation times represented by a profile of rectangles.

To find a minimal headway time the second train profile has to be shifted as close to the first profile as possible. This is where the line representing sight distance for train 2 touches the rectangle profile of train 1 [Fig. 3]. In other words: shift as long as the running time of the second train is equal to the original running time.

\subsection{ROBERTO}

The aim of ROBERTO is to compute a large number of headway times automatically for all possible pairs of trains that share one or more elements of the infrastructure. Input for the tool are running times, track element occupation times and signalling aspects relations. The computation of a headway time requires input from a microscopic infrastructure level. To realise this in a short time ROBERTO uses output from the simulation model FRISO (Flexible Rail Infrastructure Simulation model of Operations). The approach is the following:

- Build a simulation model (with FRISO) containing a part of the infrastructure network

- Define a set of trains, e.g. the trains of the timetable pattern, coming from planning systems 


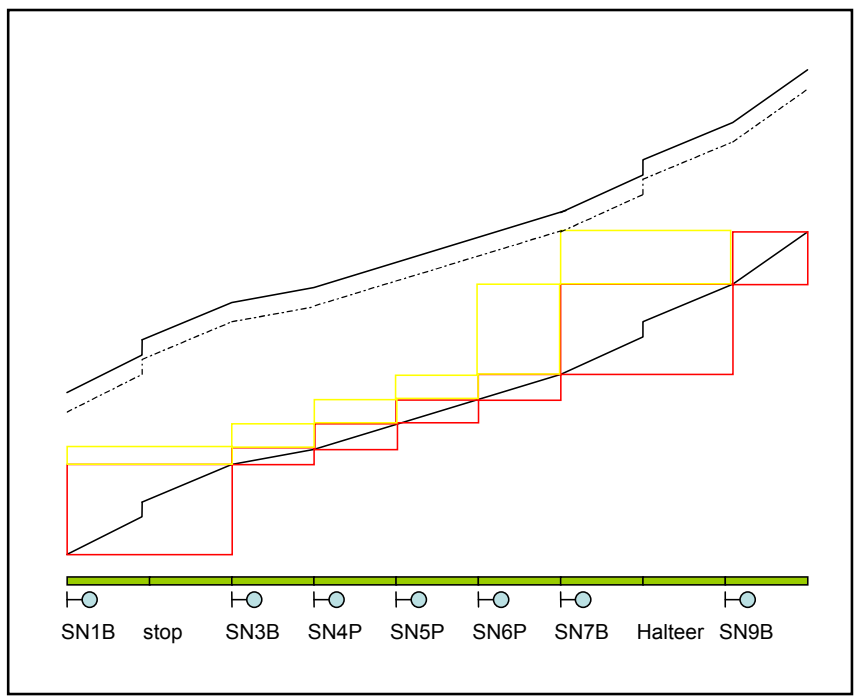

Figure 3: $\quad$ Time distance diagram with block section occupation.

- Generate the running times by single simulation runs, log train behaviour and information of the signalling system.

- $\quad$ Feed this data to ROBERTO (XML-file).

- Select ROBERTO parameters and run it.

- Analyse ROBERTO output

- Convert and feed the ROBERTO output to the planning system DONS

The data flow has the following components:

\begin{tabular}{llcc} 
IA/DONNA & FRISO & ROBERTO & DONS/DONNA \\
\hline Company & Simulation & Headway tool Timetabling \\
Databases & Model & & system \\
\hline Infrastructure & Running times & Headway times Headway times \\
Timetable & Track occupation & \multicolumn{2}{c}{ Norms, standards } \\
Routes & Signalling aspects & &
\end{tabular}

The user chooses to compute all given or a selection of trains. ROBERTO makes appropriate combinations of the trains and calculates the time differences on all shared infrastructure objects. Note that for two trains following each other more objects are shared than two trains running in opposite directions, with exception of single track use. For succeeding trains, time differences on each common signal become available. The last step is to define the critical/minimal headway time and to adjust it to the reference location of the timetable. To find a minimal headway time the second train profile has to be shifted as close to the first profile as possible without changing the original unhindered running time.

For inspection of the speed profile of a train there is a speed-time diagram, for inspection of the critical headway time and place there is a time-distance diagram 
with a visualisation of the block section occupation times (Fig. 3). The planning system DONS uses headway times on different levels. For instance, there is the most global level, meaning there is a headway time valid for any train pair in any location and there is the most detailed level where a headway time for specific trains on specific locations may be defined. In total there are 5 levels of detail. The system has a mesoscopic model for the infrastructure of the network. Each station or junction is a node and each connection is a link. Both node and links know the amount of tracks inside them and which train movements are potentially conflicting. The results of ROBERTO are based on a microscopic infrastructure model. Therefore, there is an extra step/interface which converts the critical headway times to the DONS nodes and the right level.

\section{Future work}

In a first step, the results are validated. The results and the calculation performance are promising. In a triangular part of the network between Den Bosch - Eindhoven and Tilburg (an area of approximately 60 kilometres), having 22 trains, ROBERTO finds about 1200 headway times in 20 minutes. Next step is to calculate the headway times for the main corridors and to analyse effects on capacity and punctuality performance. Finally, the tool will be connected to all planning systems, to support both timetable development and capacity allocation.

\section{References}

[1] Poort, J.P.: Limits on utilization (in Dutch). NYFER (2002)

[2] Schrijver, A., Steenbeek, A.: Timetable construction for Railned (Dienstregelingontwikkeling voor Railned). Center for Mathematics and Computer Science Amsterdam (1994)

[3] Zwaneveld, P., Dauzere-Perez, S., Van Hoesel, S., Kroon, L., Romeijn, H., Salomon, M., Ambergen, H.: Routing Trains Through Railway Stations: Model Formulation and Algorithms, Transportation Science 30 (1996) 181194

[4] Vromans, M.J.C.M...: Reliability of Railway Systems. ERIM PhD Series Research in Management 62 (2005) 56-63

[5] Kroon, L et al.: The new Dutch Timetable, the OR revolution. Interfaces (to appear 2009)

[6] Hansen, I.A., Pachl, J.: Railway Timetable \& Traffic (2008) 